\title{
Patrones de concentración social de factores de riesgo aterosclerótico y enfermedades del corazón en La Habana
}

\section{Patterns of social concentration of atherosclerosis and heart disease risk factors in La Habana province}

\author{
DrC. J orge Bacallao Gallestey,' DraC. Georgia Díaz-Perera Fernández,' DrC. \\ Eduardo Alemañy Pérez " \\ ' Centro de Investigaciones y Referencia de Aterosclerosis de La Habana (CIRAH). La \\ Habana, Cuba. \\ "Vicerrectoría Primera. Universidad de Ciencias Médicas de La Habana. La Habana, \\ Cuba.
}

\section{RESUMEN}

Objetivos: identificar factores de concentración social en la carga de morbilidad por hipertensión, diabetes tipo 2, hábito de fumar y enfermedades del corazón, y su descomponibilidad con respecto al contexto; confirmar la existencia de dimensiones no explícitas en relación con las cuales hay diferencias que se manifiestan en los factores de riesgo y en las enfermedades consecuentes de la aterosclerosis.

Métodos: se realizó un estudio transversal en 840 familias pertenecientes a 12 consultorios del médico y la enfermera de la familia. Se utilizaron indicadores objetivos de la condición socioeconómica y un indicador subjetivo basado en la percepción individual. A escala de la vivienda se calcularon densidades de morbilidad. Resultados: la desigualdad es principalmente dentro de las poblaciones atendidas por los consultorios y no entre ellos. La concentración de la carga de morbilidad es baja con respecto a indicadores objetivos y ligeramente alta con respecto a un indicador subjetivo de la condición socioeconómica.

Conclusiones: hay bajos niveles de covariación entre la dimensión socioeconómica y la dimensión salud. Se identifica una subpoblación con cargas elevadas de morbilidad, que presenta también una configuración singular de los indicadores socioeconómicos.

Palabras clave: desigualdad, índice de Theil, diabetes, hipertensión arterial, hábito de fumar, análisis de conglomerados basados en modelos. 


\section{ABSTRACT}

Objectives: to identify social concentration factors in the burden of morbidity for hypertension, type II diabetes, smoking and heart diseases, and their contextual decomposability; and to confirm the existence of latent dimensions across which there are differences in the distribution of risk factors and atherosclerosis-related diseases. Methods: a cross-sectional study was conducted in 840 families from 12 family doctor's offices. Some objective indicators of the socioeconomic condition as well as one subjective indicator based on self-assessed economic situation were used. At the household level, several indicators of morbidity density were also measured.

Results: total inequality mainly occurs within the populations cared for by the family doctor's offices and not among them. The concentration of the morbidity burden is low with respect to objective indicators, and slightly high with respect to the subjective indicator based on self-assessed economic condition.

Conclusions: the levels of co-variation between the socioeconomic and the health dimensions are low. A population segment with high levels of morbidity and singular configuration of the socioeconomic indicators was identified.

Key words: inequality, Theil index, diabetes, blood hypertension, smoking, modelbased cluster analysis.

\section{NTRODUCCI ÓN}

En relación con las enfermedades crónicas no transmisibles (ECNT), la Epidemiología moderna aún se orienta prioritariamente a la identificación de factores de riesgo. Sin embargo, cada vez se consolida más el reconocimiento de que una comprensión cabal de los determinantes de la salud a escala poblacional, en términos que van más allá de los factores de riesgo individual (y sus mediadores biológicos), exige la aplicación de una perspectiva socioecológica. Al menos dos importantes restricciones limitan aún la acción práctica de la Epidemiología contemporánea (aunque tal vez no su proyección académica): 1. Una preocupación exagerada o excluyente por los factores de riesgo individuales. 2 . Una visión temporal transversal en relación con el modo en que los sujetos cambian su condición de riesgo. ${ }^{1}$

No obstante, el contenido de la Epidemiología evoluciona continuamente, recuperando su antigua y, en cierto sentido, original visión del contexto físico y social, como determinante o factor conductor de los perfiles de salud y enfermedad. Esta evolución se manifiesta en una visión crecientemente ecológica de la salud poblacional, en el desarrollo de modelos dinámicos (a lo largo del curso de la vida) del proceso de adquisición y modificación de los riesgos, en una conceptualización más clara de la relación dialéctica entre riesgo y vulnerabilidad, y de modo general, en una extensión de los marcos de referencia espacio temporales que toman en cuenta las presiones recíprocas entre las actividades humanas y el contexto físico y social. ${ }^{2}$

En los últimos 30 años muchos estudios con nivel de anclaje individual realizados principalmente en países desarrollados, han mostrado un fuerte patrón social en la enfermedad coronaria con mayor incidencia, prevalencia y mortalidad en las clases más pobres. ${ }^{3,4}$ 
Por otra parte, otros estudios realizados a escala ecológica dan cuenta de claros gradientes de mortalidad por enfermedades coronarias en comunidades con características socioambientales diferentes. ${ }^{5,6}$

La aplicación de la modelación jerárquica ha permitido realizar estimaciones más confiables de la influencia del contexto sobre las ECNT y en particular sobre las enfermedades cardiovasculares, no solo como agentes causales directos o mediados por los factores individuales, sino también como modificadores de los efectos de estos últimos. ${ }^{7-9}$

También en los tres últimos decenios ha crecido el interés por entender, analizar y reducir las desigualdades en salud. Mientras que en algunos países europeos se ha documentado ampliamente la existencia de desigualdades en salud, ${ }^{10}$ en otros se ha transitado de la mera constatación de su existencia, al debate sobre sus causas. ${ }^{11}$

Las desigualdades en salud se reconocen cada vez más como un tema importante de la salud pública en el mundo. Esta atención internacional se hizo patente, por primera vez, en la Declaración de Alma-Atá, en 1978, cuando se formuló un pronunciamiento sobre la injusticia y la evitabilidad de las desigualdades. Poco tiempo después, el "Reporte Negro" (Black Report) ${ }^{10}$ del Reino Unido se convirtió en el primer estudio que incluyó explícitamente el tema de la desigualdad sanitaria, y despertó el interés por su estudio en muchos otros países.

La mala salud de los pobres, las grandes desigualdades sanitarias entre los países y el gradiente social de salud dentro de estos, se deben a una distribución desigual en el nivel correspondiente, del poder, los ingresos, los bienes y los servicios, y a las consiguientes injusticias que afectan las condiciones de vida de la población en términos de acceso a atención sanitaria de calidad, escolarización, educación, condiciones de trabajo y tiempo libre, calidad de la vivienda, y la posibilidad de alcanzar todo el potencial de desarrollo. Esa distribución desigual de las condiciones de salud no es, en ningún caso, un fenómeno natural. Los determinantes estructurales y las condiciones de vida en su conjunto constituyen sus determinantes sociales. ${ }^{12-20}$

Cuba sufrió a partir de los años 1989-90, tras el derrumbe de la Unión Soviética y el campo socialista, la severa crisis económica conocida como "período especial". A ella se unió el reforzamiento del bloqueo económico y comercial de los Estados Unidos de Norteamérica.

La crisis y el reajuste económico que marcaron a la sociedad cubana de los 90, han tenido entre sus efectos más relevantes, una reconfiguración de la estructura social que incluye procesos de emergencia y desaparición de diferentes grupos sociales, el fortalecimiento y el debilitamiento económico de otros, el ensanchamiento de las distancias sociales y las desigualdades, y la aparición de sectores sociales en riesgo y de estratos en situación de ventaja relativa. ${ }^{21-25}$

Los resultados que se exponen en el presente artículo forman parte de un proyecto de investigación del Centro de Investigaciones y Referencia de Aterosclerosis de La Habana ( $\mathrm{CIRAH}$ ) cuyo propósito es estudiar los factores de riesgo individual y los determinantes contextuales de las enfermedades consecuentes de la aterosclerosis. Este trabajo dirige su atención al estudio de la agregación social de la carga de morbilidad con respecto a la hipertensión, la diabetes y las enfermedades del corazón, y a la frecuencia relativa de fumadores, en relación con varios marcadores objetivos, y con un indicador subjetivo de la condición socioeconómica. El análisis, sin antecedentes en el país bajo este enfoque metodológico, tiene una doble finalidad: por un lado, confirmar la conjetura de que en Cuba hay una baja covariación entre la 
dimensión socioeconómica y la dimensión salud, y por el otro, que pese a esa baja covariación hay dimensiones no explícitas en relación con las cuales existen diferencias que se manifiestan en los factores de riesgo y en las enfermedades consecuentes de la aterosclerosis, probablemente como caso particular de otras diferencias en salud.

\section{MÉTODOS}

Se realizó un estudio transversal en 840 familias pertenecientes a 12 consultorios del médico y la enfermera de la familia (CMF). La selección se llevó a cabo en dos etapas: en la primera se seleccionaron los consultorios mediante un procedimiento discrecional (no probabilístico) en función de criterios de factibilidad para la recolección de la información, en la segunda etapa se seleccionaron las familias mediante muestreo simple aleatorio. La unidad de análisis es la familia, lo cual explica que los indicadores de salud no se expresen en términos de prevalencias, sino de densidades de morbilidad, que se definen como el cociente entre la cantidad de personas afectadas y el total de susceptibles en cada familia, en el grupo de edad de 20 años o más. Las variables que se utilizaron fueron las siguientes:

Dependientes: densidades de morbilidad por hipertensión, diabetes mellitus, enfermedades del corazón (cardiopatía isquémica, insuficiencia cardiaca, arritmia e infarto del miocardio) y hábito de fumar, todas ellas calculadas como se acaba de indicar.

No se practicaron mediciones individuales (no se hicieron con respecto a las variables que se emplean en el presente estudio, aunque sí en relación con las variables del proyecto, del cual forma parte esta investigación), salvo para la tensión arterial . En todos los demás casos, la condición de cada sujeto se obtuvo a partir de la dispensarización familiar y del testimonio de los encuestados.

La condición de hipertenso podía modificarse si un "no hipertenso" de acuerdo al criterio anterior, presentaba cifras de tensión arterial superiores a $140 \mathrm{mmHg}$ para la tensión sistólica o a $90 \mathrm{mmHg}$ para la diastólica, replicadas al día siguiente de la medición original.

Independientes:

- Escolaridad: 0. analfabeto (a); 1. primaria; 2. secundaria; 3: técnico medio o preuniversitario; 4 . universitaria.

La máxima del hogar es la que ostenta la persona con la más alta escolaridad reportada.

- Equipos de primera necesidad: el número de equipos en la vivienda (a partir de declaración y comprobación del encuestador) del listado siguiente: radio, plancha, ventilador, televisor, refrigerador y olla de presión.

- Equipos de segunda necesidad: el número de equipos en la vivienda (a partir de declaración y comprobación del encuestador) del listado siguiente: grabadora, batidora, aire acondicionado, tocadiscos, horno microonda, ventilador, video, máquina de coser, freezer, calentador de agua y cualquier otro no incluido en el listado de primera necesidad. 
- Situación económica subjetiva de acuerdo a la percepción de cada uno de los miembros de la vivienda. 1 . muy buena; 2 . buena; 3. regular; 4. mala; 5 . muy mala. Se tomó, para este estudio, la percepción más desfavorable, entre todas las personas encuestadas en la vivienda.

Se calculó el índice de Theil, ${ }^{26}$ que es una medida de desigualdad basada en la noción de entropía, con respecto a la hipertensión, la diabetes tipo 2, el hábito de fumar y las enfermedades del corazón. Este índice se utiliza como medida de desigualdad en salud, y no como indicador de las desigualdades asociadas a un dominio socioeconómico.

El recuadro y las expresiones subsiguientes definen al índice de Theil:

Recuadro. Distribución de la población y de la carga de enfermedad en K grupos o clases

\begin{tabular}{|c|c|c|}
\hline Clases & Población & $\begin{array}{c}\text { Variable } \\
\text { de salud }\end{array}$ \\
\hline 1 & $\Pi_{1}$ & $\mathrm{P}_{1}$ \\
\hline 2 & $\Pi_{2}$ & $\mathrm{P}_{2}$ \\
\hline$\cdot$ & $\cdot$ & $\cdot$ \\
\hline$\cdot$ & $\cdot$ & $\cdot$ \\
\hline$\cdot$ & $\cdot$ & $\cdot$ \\
\hline $\mathrm{K}$ & $\Pi_{\mathrm{K}}$ & $\mathrm{PK}_{\mathrm{K}}$ \\
\hline Total & 1 & 1 \\
\hline
\end{tabular}

$K$ es el número de unidades geodemográficas. En este caso $K=840$ familias. En cada una de ellas, la población es $N_{k}$, y la cantidad de enfermos $S_{K}$, de modo que:

$$
\begin{gathered}
N=\sum_{i=1}^{K} N_{i} \\
S=\sum_{i=1}^{K} S_{i} \\
\bar{\pi}_{i}=\frac{N_{i}}{N} ; \quad P_{i}=\frac{S_{i}}{S}
\end{gathered}
$$

El índice de Theil es un modo particular de medir la discrepancia entre las sucesiones $\pi_{\mathrm{i}}$ y $\mathrm{P}_{\mathrm{i}}$ que son, en rigor, dos funciones de probabilidad, ya que:

$$
\sum_{j=i}^{K} \pi_{j}=\sum_{j=1}^{K} P_{j}=1
$$


El índice de Theil se define del siguiente modo:

$$
I_{T}=\sum_{i=1}^{K} P_{i} \ln \left(\frac{P_{i}}{\pi_{i}}\right)
$$

Una de sus propiedades más interesantes es que puede descomponerse aditivamente en un Theil "entre" y un Theil "dentro" (en este caso "entre consultorios" y "dentro de los consultorios") en donde estos componentes expresan, respectivamente, la contribución relativa a la desigualdad total. Se aplicó también la llamada "equivalencia de entropía", ${ }^{26}$ que hace posible equiparar la desigualdad en el total de clases (consultorios en este caso particular) a la desigualdad de una población hipotética de dos clases lo cual aporta a la medición una interpretación basada en el potencial de cambio necesario para alcanzar la igualdad.

Se calculó la correlación de la desigualdad con las medidas de densidad agregadas a nivel de consultorio.

Se calculó también el índice de concentración de la carga de morbilidad por hipertensión, diabetes mellitus tipo II, y enfermedades del corazón, y de la frecuencia relativa de fumadores, en relación con los marcadores objetivos de la condición socioeconómica y con la medida subjetiva basada en la autopercepción de las personas entrevistadas en cada vivienda. El índice de concentración es una medida de desigualdad social en salud, que se deriva de la llamada "curva de concentración" cuya racionalidad es similar a la del indice de Gini, la métrica más conocida de desigualdad en relación con el ingreso. Para construir la curva de concentración, la población se ordena de peor a mejor según un indicador socioeconómico elegido, y se obtiene la carga acumulada en la variable de salud para distintos valores de la proporción de población acumulada. El índice de concentración toma valores entre - 1 $y+1$. Convencionalmente los valores positivos corresponden a curvas de concentración que se encuentran por debajo de la diagonal principal (que representa la situación de total igualdad) y los valores negativos, a curvas que se encuentran por encima de dicha diagonal. En condiciones de desigualdad, el índice toma valores positivos cuando se trata de acceso a servicios de salud, y valores negativos cuando se trata de carga de enfermedad. ${ }^{27}$

Por último, se aplicó el análisis de conglomerados basados en modelos, ${ }^{28}$ para identificar subpoblaciones con diferentes perfiles epidemiológicos para los factores de riesgo y las enfermedades consecuentes de la aterosclerosis. En 46 familias del total de 840 estudiadas en 12 consultorios de 6 áreas de salud, algunas de las variables estaban ausente, por lo que el análisis de conglomerados basado en modelos se concentró en las 794 familias con datos completos.

\section{RESULTADOS}

En la tabla 1 se observa que los mayores niveles de desigualdad ocurren con respecto a las enfermedades del corazón y los menores con respecto a la hipertensión. Es importante destacar, que lo que se mide en el presente caso con el índice de Theil no es desigualdad social, sino desigualdad en salud, ya que no hay ningún vínculo explícito entre salud y condición socioeconómica en el cálculo del índice. A diferencia de lo que ocurre con las aplicaciones estrictamente económicas del índice de Theil, la 
mayor concentración de la carga de morbilidad se asocia con las menores prevalencias. Hay dos hechos particularmente interesantes que se advierten en esta tabla. El primero es que la mayor desigualdad corresponde a las entidades con menor densidad de morbilidad. El segundo, relacionado con el anterior, es que entre la densidad de morbilidad y la desigualdad hay correlaciones negativas para todas las entidades estudiadas y altas para todas, con la única excepción de la hipertensión. El hecho es de gran trascendencia desde el punto de vista metodológico, porque sugiere que las acciones dirigidas a la disminución de la frecuencia relativa de una enfermedad, pueden ir acompañadas de un incremento en la desigualdad, independientemente del criterio de medición. Otros autores ya habían constatado una relación parecida al utilizar indicadores simples de la desigualdad como el cociente de tasas, que tiende a aumentar a medida que dos subpoblaciones desiguales disminuyen sus tasas de prevalencia. A este hecho, en apariencia sorprendente, se le conoce como "paradoja del suceso raro. "29,30

Tabla 1. Densidad familiar de diabetes, hipertensión, hábito de fumar y enfermedades del corazón y valores del índice de Theil por consultorios del médico de familia

\begin{tabular}{|c|c|c|c|c|c|c|c|c|}
\hline \multirow{2}{*}{$\begin{array}{l}\text { No. } \\
\text { CMF }\end{array}$} & \multicolumn{2}{|c|}{ Diabetes } & \multicolumn{2}{|c|}{ HTA } & \multicolumn{2}{|c|}{ Hábito de fumar } & \multicolumn{2}{|c|}{ Enf. del corazón } \\
\hline & $\begin{array}{c}\text { Densidad } \\
\text { (\%) }\end{array}$ & Theil & $\begin{array}{c}\text { Densidad } \\
\text { (\%) }\end{array}$ & Theil & $\begin{array}{c}\text { Densidad } \\
\text { (\%) }\end{array}$ & Theil & $\begin{array}{c}\text { Densidad } \\
(\%)\end{array}$ & Theil \\
\hline 1 & 10,0 & 1,76 & 31,6 & 0,58 & 18,8 & 0,79 & 13,5 & 0,89 \\
\hline 2 & 5,8 & 1,60 & 34,4 & 0,40 & 31,9 & 0,75 & 11,8 & 1,56 \\
\hline 3 & 9,1 & 1,36 & 29,1 & 0,76 & 24,9 & 0,74 & 13,3 & 1,38 \\
\hline 4 & 13,4 & 1,44 & 25,1 & 0,55 & 25,8 & 1,03 & 5,2 & 2,29 \\
\hline 5 & 11,5 & 1,39 & 34,0 & 0,54 & 23,6 & 1,20 & 6,4 & 1,55 \\
\hline 6 & 8,6 & 1,65 & 31,1 & 0,59 & 21,7 & 0,65 & 11,3 & 1,37 \\
\hline 7 & 8,3 & 1,55 & 21,5 & 0,53 & 31,4 & 0,90 & 4,6 & 2,18 \\
\hline 8 & 5,7 & 1,58 & 26,4 & 0,56 & 18,1 & 1,03 & 6,4 & 1,45 \\
\hline 9 & 10,6 & 1,58 & 29,6 & 0,56 & 21,2 & 1,03 & 10,5 & 1,39 \\
\hline 10 & 9,3 & 1,72 & 26,8 & 0,58 & 19,6 & 1,02 & 8,9 & 2,03 \\
\hline 11 & 5,6 & 2,17 & 30,8 & 0,64 & 26,5 & 0,64 & 4,0 & 2,54 \\
\hline 12 & 5,6 & 1,95 & 27,8 & 0,78 & 26,8 & 0,82 & 8,8 & 1,48 \\
\hline Total & 8,6 & 1,65 & 29,0 & 0,71 & 24,2 & 0,77 & 8,7 & 1,57 \\
\hline$P$ & \multicolumn{2}{|c|}{ - 0,55 } & \multicolumn{2}{|c|}{$-0,04$} & \multicolumn{2}{|c|}{$-0,32$} & \multicolumn{2}{|c|}{$-0,79$} \\
\hline
\end{tabular}

p: Coeficiente de correlación por rangos de Spearman.

La tabla 2 muestra que la desigualdad total es, casi toda, a expensas de la desigualdad dentro. Esto quiere decir que la mayor proporción de desigualdad no depende de factores propios de los consultorios que diferencian a unos de otros, sino de factores internos. La expresión "factores propios de los consultorios", no alude a las características de los consultorios, sino a características de las poblaciones a las que el consultorio da cobertura.

No parece haber una agregación espacial en relación con las poblaciones servidas por los consultorios, sino que las fuentes de desigualdad se encuentran de manera abrumadoramente mayoritaria, en el interior de estos (menos de $5 \%$ de la desigualdad total en todos los casos es desigualdad "entre" consultorios).

Para cada valor del índice se ha incluido entre paréntesis la equivalencia de entropía, ${ }^{26}$ que es un recurso que facilita la interpretación de los índices basados en esta noción. Las cifras entre paréntesis corresponden a una sociedad hipotética 
dividida en dos clases, cuya desigualdad sería equivalente (en el sentido de la entropía) a la que es objeto de medición. Por ejemplo, a la desigualdad total de 0,71 con respecto a la hipertensión, corresponde una equivalencia de entropía de 0,22; lo que quiere decir que la desigualdad presente en la población estudiada es equivalente a la que exhibiría una hipotética sociedad dividida en dos clases en la que una de ellas concentrase el $78 \%$ de la población y el $22 \%$ de la carga de morbilidad, y la otra, el $22 \%$ de la población y el $78 \%$ de la carga de morbilidad. Para alcanzar la completa igualdad, la carga de morbilidad por hipertensión, tendría que redistribuirse en $28 \%$.

En congruencia con el patrón de distribución de la desigualdad, la tabla 2 muestra que el potencial de redistribución de la carga de morbilidad entre consultorios es muy pequeño, como expresión de los bajos niveles de desigualdad "entre". Sin embargo, el potencial distributivo es alto "dentro", lo que apunta a la necesidad de identificar los factores que a escala familiar explican las diferencias.

Tabla 2. Descomposición aditiva del índice de Theil para la diabetes, la hipertensión arterial, el hábito de fumar y las enfermedades del corazón

\begin{tabular}{|l|c|c|c|c|}
\hline \multicolumn{4}{|c}{} & \multicolumn{2}{|c|}{ Theil Total= Theil entre + Theil dentro } \\
& Theil Total & Theil entre & Theil dentro & \% entre \\
\hline Diabetes & $1,65(0,11)$ & $0,04(0,43)$ & $1,61(0,11)$ & 2,3 \\
\hline HTA & $0,71(0,22)$ & $0,01(0,46)$ & $0,70(0,22)$ & 1,1 \\
\hline Hábito de fumar & $0,77(0,21)$ & $0,02(0,45)$ & $0,75(0,21)$ & 2,5 \\
\hline Enf. del corazón & $1,57(0,12)$ & $0,06(0,41)$ & $1,51(0,12)$ & 4,0 \\
\hline
\end{tabular}

Entre paréntesis, la equivalencia de entropía.

Fuente: base de datos.

Cabe señalar, sin embargo, que si bien la noción de redistribución es perfectamente natural en relación con los recursos o los servicios de salud, resulta mucho más contraria a la intuición con respecto a la carga de morbilidad. Dada una carga de morbilidad, cualquier cambio tendría que ser concebido a expensas de una reducción de dicha carga y no de una redistribución entre individuos o grupos poblacionales.

La tabla 3 contiene la distribución de las densidades de morbilidad para diabetes, hipertensión, hábito de fumar y enfermedades del corazón, de acuerdo a las clases de dos indicadores objetivos de la condición socioeconómica (escolaridad y equipos de segunda necesidad) y a su percepción subjetiva. Solo en relación con esta última se aprecia un gradiente claro, aunque muy moderado de las densidades de morbilidad.

La disociación entre la dimensión salud y la dimensión socioeconómica, se aprecia en la tabla 4, que contiene los valores del índice de concentración. Los valores más altos son los que se obtienen con respecto al indicador subjetivo de la condición económica, pero son en general valores pequeños. Todos los valores son negativos lo que indica mayor densidad en las clases definidas por los valores más desfavorables de las variables.

En la búsqueda de dimensiones de agregación no directamente observables de las enfermedades asociadas a la aterosclerosis, se llevó a cabo un análisis de conglomerados basados en modelos, ${ }^{28}$ cuyos resultados se resumen en las tablas de la 5 a la 7 . 
Tabla 3. Densidad familiar de diabetes, hipertensión, hábito de fumar y enfermedades del corazón por categorías de escolaridad, equipos de segunda necesidad y situación económica percibida

\begin{tabular}{|c|c|c|c|c|c|}
\hline $\begin{array}{l}\text { Indicadores } \\
\text { socioeconómicos }\end{array}$ & Categorías & Diabetes & Hipertensión & $\begin{array}{l}\text { Hábito de } \\
\text { fumar }\end{array}$ & $\begin{array}{c}\text { Enfermedades } \\
\text { del corazón }\end{array}$ \\
\hline \multirow{3}{*}{ Escolaridad } & Primaria & 0,08 & 0,25 & 0,24 & 0,09 \\
\hline & Secundaria & 0,09 & 0,29 & 0,24 & 0,08 \\
\hline & $\begin{array}{l}\text { Preuniversitaria } \\
\text { o más }\end{array}$ & 0,08 & 0,26 & 0,22 & 0,07 \\
\hline \multirow{3}{*}{$\begin{array}{l}\text { Equipos de segunda } \\
\text { necesidad }\end{array}$} & 0 & 0,08 & 0,28 & 0,26 & 0,09 \\
\hline & $1-2$ & 0,07 & 0,26 & 0,21 & 0,09 \\
\hline & $3-5$ & 0,10 & 0,27 & 0,19 & 0,07 \\
\hline \multirow{3}{*}{$\begin{array}{l}\text { Percepción subjetiva } \\
\text { de la situación } \\
\text { económica }\end{array}$} & Mala o muy mala & 0,12 & 0,32 & 0,30 & 0,13 \\
\hline & Regular & 0,08 & 0,27 & 0,24 & 0,08 \\
\hline & $\begin{array}{l}\text { Excelente } \\
\text { o buena }\end{array}$ & 0,07 & 0,23 & 0,17 & 0,08 \\
\hline
\end{tabular}

La tabla 5 revela la existencia de tres conglomerados, uno de los cuales, al que se ha llamado "Conglomerado 0", está formado por 90 familias que representan 11,3\% del total. ${ }^{*}$ Aunque no se advierten diferencias ostensibles entre los conglomerados en las variables socioeconómicas, hay un patrón sistemático que se aprecia a simple vista en la propia tabla 5: el "Conglomerado 0" está compuesto por núcleos familiares que en promedio: tienen un menor número de integrantes, más baja escolarización, un número discretamente menor de equipos de primera necesidad, y una percepción más desfavorable de su situación económica. La variable "situación económica subjetiva" tiene una polaridad diferente del resto de las variables, por lo cual los valores más altos corresponden a la percepción más desfavorable.

Tabla 5. Valores promedios y desviación estándar (Medias y DE) de las variables socioeconómicas de las subpoblaciones

\begin{tabular}{|c|c|c|c|c|c|c|}
\hline & & $\begin{array}{l}\text { Personas } \\
\text { por } \\
\text { vivienda }\end{array}$ & $\begin{array}{c}\text { Máxima } \\
\text { escolaridad } \\
\text { en el hogar }\end{array}$ & $\begin{array}{l}\text { Equipos } \\
\text { de } \\
\text { segunda } \\
\text { necesidad }\end{array}$ & $\begin{array}{l}\text { Equipos } \\
\text { de } \\
\text { primera } \\
\text { necesidad }\end{array}$ & $\begin{array}{l}\text { Situación } \\
\text { económica } \\
\text { subjetiva }\end{array}$ \\
\hline \multirow{2}{*}{$\begin{array}{l}\text { Conglomerado } 0 \\
(n=90 ; 11,3 \%)\end{array}$} & Media & 2,87 & 7,51 & 1,34 & 5,58 & 3,27 \\
\hline & $\begin{array}{l}\text { Desviación } \\
\text { estándar }\end{array}$ & 1,16 & 3,72 & 1,89 & 0,76 & 1,03 \\
\hline \multirow{2}{*}{$\begin{array}{l}\text { Conglomerado } 1 \\
(n=430 ; 54,2 \%)\end{array}$} & Media & 3,16 & 10,23 & 1,23 & 5,68 & 3,03 \\
\hline & $\begin{array}{l}\text { Desviación } \\
\text { estándar }\end{array}$ & 1,29 & 2,99 & 1,38 & 0,64 & 0,22 \\
\hline \multirow{2}{*}{$\begin{array}{l}\text { Conglomerado } 2 \\
(n=274 ; 34,5 \%)\end{array}$} & Media & 3,08 & 9,61 & 2,14 & 5,75 & 2,27 \\
\hline & $\begin{array}{l}\text { Desviación } \\
\text { estándar }\end{array}$ & 1,21 & 4,28 & 1,98 & 0,59 & 0,82 \\
\hline
\end{tabular}

La tabla 6 revela que, aun después de ajustar para la edad, las densidades de morbilidad son más altas en el Conglomerado 0 que en el resto. Por último, la tabla 7 contiene los resultados del ajuste de un modelo lineal, que tiene el propósito de evaluar el efecto "conglomerado" sobre las densidades de morbilidad, luego de ajustar para la edad y para el resto de las variables socioeconómicas. Para todas las densidades hay un efecto altamente significativo del conglomerado. 
Tabla 6. Medias ajustadas para la edad edn las densidades de diabetes, hipertensión y hábito de fumar según conglomerados

\begin{tabular}{|c|c|c|c|c|}
\hline \multirow{2}{*}{ Densidad } & \multirow{2}{*}{ Conglomerados } & \multirow{2}{*}{ Media } & \multicolumn{2}{|c|}{$95 \%$ Intervalo de Confianza } \\
\hline & & & Límite inferior & Límite superior \\
\hline \multirow{3}{*}{$\begin{array}{l}\text { Densidad } \\
\text { de diabéticos }\end{array}$} & 0 & 0,19 & 0,15 & 0,22 \\
\hline & 1 & 0,07 & 0,05 & 0,08 \\
\hline & 2 & 0,07 & 0,05 & 0,09 \\
\hline \multirow{3}{*}{$\begin{array}{l}\text { Densidad } \\
\text { de hipertensos }\end{array}$} & 0 & 0,41 & 0,36 & 0,46 \\
\hline & 1 & 0,26 & 0,23 & 0,28 \\
\hline & 2 & 0,23 & 0,20 & 0,26 \\
\hline \multirow{3}{*}{$\begin{array}{l}\text { Densidad } \\
\text { de fumadores }\end{array}$} & 0 & 0,36 & 0,30 & 0,41 \\
\hline & 1 & 0,23 & 0,20 & 0,26 \\
\hline & 2 & 0,16 & 0,13 & 0,20 \\
\hline \multirow{3}{*}{$\begin{array}{l}\text { Densidad } \\
\text { de enfermos del corazón }\end{array}$} & 0 & 0,14 & 0,10 & 0,18 \\
\hline & 1 & 0,07 & 0,05 & 0,09 \\
\hline & 2 & 0,05 & 0,05 & 0,09 \\
\hline
\end{tabular}

Tabla 7. Resultados del modelo lineal ( $\mathrm{F}$ $p$ )* para evaluar las diferencias entre conglomerados, ajustadas para variables individuales

\begin{tabular}{|c|c|c|c|c|c|c|c|c|}
\hline \multirow[t]{2}{*}{ Variables } & \multicolumn{2}{|c|}{$\begin{array}{c}\text { Densidad } \\
\text { de diabéticos }\end{array}$} & \multicolumn{2}{|c|}{$\begin{array}{l}\text { Densidad } \\
\text { de hipertensos }\end{array}$} & \multicolumn{2}{|c|}{$\begin{array}{c}\text { Densidad } \\
\text { de fumadores }\end{array}$} & \multicolumn{2}{|c|}{$\begin{array}{l}\text { Densidad } \\
\text { de enfermos } \\
\text { del corazón }\end{array}$} \\
\hline & $\mathrm{F}$ & $p$ & $\mathrm{~F}$ & $p$ & $\mathrm{~F}$ & $p$ & $\mathrm{~F}$ & $p$ \\
\hline Edad & 38,19 & 0,00 & 165,17 & 0,00 & 0,87 & 0,34 & 68,41 & 0,00 \\
\hline Personas por vivienda & 0,02 & 0,88 & 3,11 & 0,07 & 0,00 & 0,99 & 0,02 & 0,88 \\
\hline $\begin{array}{l}\text { Máxima escolaridad } \\
\text { en el hogar }\end{array}$ & 0,04 & 0,82 & 2,64 & 0,10 & 4,57 & 0,03 & 0,03 & 0,86 \\
\hline Condición económica subjetiva & 1,08 & 0,29 & 1,99 & 0,15 & 1,22 & 0,26 & 1,51 & 0,22 \\
\hline Equipos & 7,79 & 0,00 & 0,12 & 0,72 & 1,04 & 0,30 & 1,27 & 0,26 \\
\hline Conglomerado & 18,47 & 0,00 & 16,71 & 0,00 & 11,73 & 0,00 & 5,85 & 0,00 \\
\hline
\end{tabular}

*F: estadístico de Fisher, $p$ : valor de la significación estadística.

\section{DISCUSIÓN}

Hay evidencia abrumadora de que los factores sociales tienen un gran impacto sobre la salud. ${ }^{31}$ Las enfermedades no se distribuyen aleatoriamente en la sociedad, los individuos en posiciones de desventaja socioeconómica son más susceptibles a cambios desfavorables en sus perfiles de riesgo. Las explicaciones tentativas de este hecho, sugieren que las desigualdades sociales aumentan la susceptibilidad a sufrir enfermedades y disminuyen la resistencia a sus posibles efectos, así como también generan una menor conciencia de riesgo, y consecuentemente menor motivación por las conductas responsables, la adopción de medidas de autocuidado y el acceso a la atención médica oportuna. Todo ello porque la condición socioeconómica relativa tiene tanto o mayor impacto sobre la salud que la propia condición socioeconómica en términos absolutos. ${ }^{32}$ 
Las desigualdades en materia de salud tanto dentro como entre los países se manifiestan intergeneracionalmente y tienen su origen en desigualdades de oportunidades a lo largo del curso de la vida. ${ }^{33}$

En la búsqueda de factores de agregación de la carga de morbilidad, los resultados de la presente investigación muestran, en primer lugar, que la carga de morbilidad por diabetes, hipertensión, hábito de fumar y enfermedades del corazón, no se distribuye homogéneamente en la población. No obstante, la agregación no es territorial, como lo demuestra el hecho de que un porcentaje abrumadoramente alto de la desigualdad ocurre "dentro" de la población cubierta por los consultorios, y no "entre las poblaciones", lo que se pone de manifiesto a partir de la propiedad de descomponibilidad del índice de Theil que se empleó como métrica de las desigualdades en salud.

Estos resultados se deben muy probablemente, a la gran homogeneidad en términos de salud que ha alcanzado la sociedad cubana a partir de las políticas públicas basadas en un activismo centralizado que opera a través de un sistema único de salud. En la Cuba anterior a 1959, y en otros países de la Región, hay una gran estratificación social en la distribución poblacional de los grandes asentamientos urbanos, que se manifiesta también en los indicadores de salud. La equidad, la igualdad de derechos y la no exclusión del sistema de salud cubano borraron esas diferencias, que emergen sin embargo con respecto a otros factores de estratificación no explícitos, que es importante identificar, y que no coinciden con los criterios habituales (género, color de la piel, ingreso, escolaridad, ocupación y otros) que sí son fuente de desigualdad en otros países.

Los determinantes contextuales, por tanto, no hay que buscarlos en los factores que determinan las heterogeneidades territoriales, sino en otros ejes clasificatorios que se hacen patentes dentro de los propios territorios examinados.

Un resultado colateral, aunque de gran trascendencia práctica de este estudio es la alta asociación negativa entre la carga de morbilidad y la desigualdad en salud. Esta asociación negativa, identificada por otros autores al utilizar medidas relativas de desigualdad, ${ }^{29-30}$ se manifiesta también con las métricas basadas en la noción de entropía.

Finalmente, no hay indicios de agregación geográfica de la carga de morbilidad por diabetes, hipertensión, hábito de fumar y enfermedades del corazón. La mayor proporción de variación ocurre dentro de las poblaciones atendidas por los consultorios y no entre ellas, lo cual indica que las desigualdades en los factores de riesgo y en las enfermedades asociadas a la aterosclerosis ocurren a niveles de desagregación finos, que no tienen una expresión territorial.

Se ha confirmado la expectativa de una baja asociación entre los indicadores de salud y varios indicadores de la condición socioeconómica, y se ha constatado una moderada asociación entre aquellos y la percepción subjetiva de la condición económica. El hecho, si se replica en otros proyectos de investigación futuros, confirmaría el papel relevante de la condición relativa -tanto o más que la absolutacomo determinante de la salud. Este estudio aporta indicios en tal sentido, aunque es imposible precisar los roles relativos de causa y efecto en dicha asociación.

De especial interés es la identificación de una subpoblación con perfiles elevados de riesgo. Estos perfiles no podrían explicarse mediante ninguno de los indicadores socioeconómicos tomados aisladamente, y muy probablemente tampoco, mediante otros criterios de estratificación que sí operan en otros escenarios internacionales; representan tal vez una característica singular del país, que tiene, no obstante, un 
gran significado en términos de los criterios de focalización de las políticas de salud. En general esta subpoblación se caracteriza por familias pequeñas, con una escolaridad discretamente más baja, con edad promedio ligeramente mayor y, especialmente, por una percepción más desfavorable de su propia condición económica. No obstante, ninguno de estos factores, tomado aisladamente alcanza para explicar las diferencias en las cargas de morbilidad.

\section{REFERENCI AS BI BLI OGRÁFI CAS}

1. Susser M. Does risk factor epidemiology put epidemiology at risk? Peering into the future. J Epidemiol Community Health. 1998; 52:608-11.

2. Rothman KJ, Poole C. Our conscientious objection to the epidemiology wars. J Epidemiol Community Health. 1998; 52:1-2.

3. Lynch J, Davey Smith G, Harper S. Explaning the social gradient in coronary heart disease: comparing relative and absolute risk approaches. J Epidemiol Community Health. 2006; 60:436-41.

4. Alboni P, Amadei A, Scarfo S. In industrialized nations a low socioeconomic status represents an independent predictor of mortality in patients with acute myocardial infarction. Ital Heart J. 2003; 4:551-8.

5. Wing S, Casper M, Davis W. Trends in geographic inequality of cardiovascular disease mortality in the United States, 1962-1982. Soc Sci Med. 1990; 30:261-6.

6. Logue EE, Jarjoura D. Modeling heart disease mortality with census tract rates and social class mixtures. Soc Sci Med. 1990; 31:545-50.

7. Diez-Roux AV, Nieto FJ, Muntaner C. Neighborhood environments and coronary heart disease: a multilevel analysis. Am J Epidemiol. 1997; 146:48-63.

8. Jackson $\mathrm{CH}$, Richardson S, Best NG. Studying place effects on health by synthetizing individual and area-level outcomes. Soc Sci Med. 2008; 7: 1995-2006.

9. Backlund E, Rowe G, Lynch J, Wolfson MC, Kaplan GA, Sorlie PD. Income inequality and mortality: a multilevel prospective study of 521248 individuals in 50 US states. Internat J Epidemiol. 2007; 36:5906.

10. Townsend P, Davidson N. The Black Report. London: Penguin Books; 1992.

11. Pickett KE, Dorling D. Against the organization of misery? The Marmot Review of health inequalities. Soc Sci Med. 2010; 71:1231-3.

12. Comisión Económica para América Latina y el Caribe. Panorama social de América Latina. Documento informativo [Internet]. CEPAL: Comisión; 2010 [citado 18 Feb 2011]. Disponible en: http://www.eclac.org

13. Paes de Barros R. Measuring Inequality of Opportunities in Latin America and the Caribbean. The International Bank for Reconstruction and Development [Internet]. The World Bank: The International Bank; 2009 [cited 2011 Abr 18]. Available from: http://siteresources. worldbank.org 
14. Santos H. Relación entre la pobreza, iniquidad y exclusión social con las enfermedades de alto costo en México. Rev Cubana Salud pública [Internet]. 2006 jun [citado 18 Feb 2011]; 32(2). Disponible en:

http: //scielo.sld.cu/scielo. php?script=sci_arttext\&pid=S0864-

34662006000200007\&lng=es

15. Programa de Naciones Unidas para el Desarrollo. Informe sobre Desarrollo Humano. La verdadera riqueza de las naciones: Caminos al desarrollo humano [Internet]. NN. UU.: PNUD; 2010 [citado 27 Mar 2011]. Disponible en: http://hdr.undp.org/es/informes/mundial/idh2010/capitulos/es/

16. Safaei J. Desigualdades en salud relacionada con el ingreso global. Medicina Social. $2007 ; 2(1): 21-36$.

17. Comisión Organización Mundial de la Salud sobre Determinantes Sociales de la Salud [Internet]. OMS: Comisión; 2008 [citado 27 Mar 2011]. Disponible en: http://www.who.int/social_determinants/final_report/media/csdh_report_wrs_es.pdf

18. Rojas F. Las crisis y la salud. Rev Cubana Salud Pública [Internet]. 2009 Mar [citado 18 Feb 2011]; 35(1). Disponible en:

http://scielo.sld.cu/scielo.php?script=sci_arttext\&pid=S086434662009000100002\&lng=es

19. Guthrie LC, Butler SC, Ward MM. Time perspective and socioeconomic status: A link to socioeconomic disparities in health? Soc Sc Med. 2009; 68:2145-51.

20. Alazraqui M, Mota E, Spinelli H, Guevel C. Desigualdades en salud y desigualdades sociales: un abordaje epidemiológico en un municipio urbano de Argentina. Rev Panam Salud Pública. 2007;21(1): 1-10.

21. Martínez S. Ideales de salud y vida. Rev Cubana Salud Pública [Internet]. 2006 Sep [citado 18 Feb 2011]; 32(3). Disponible en: http://scielo.sld.cu/scielo.php?script=sci_arttext\&pid=S0864$\underline{34662006000300001 \& \operatorname{lng}=\mathrm{es}}$

22. Álvarez Pérez AG, García Fariñas A, Bonet Gorbea M. Pautas conceptuales y metodológicas para explicar los determinantes de los niveles de salud en Cuba. Rev Cubana Salud Pública [Internet]. 2007 J un [citado 18 Feb 2011]; 33(2). Disponible en: http://scielo.sld.cu/scielo.php?script=sci_arttext\&pid=S0864$\underline{34662006000300001 \& \operatorname{lng}=\mathrm{es}}$

23. Díaz-Perera G, Alemañy E. Enfoque de la desigualdad social en periodo de crisis: experiencia cubana. Rev Habanera Ciencias Méd [Internet]. 2011 Mar [citado 27 Mar 2011]; 10(1). Disponible en:

http://scielo.sld.cu/scielo.php?script=sci_arttext\&pid=S1729-

$\underline{519 \times 2011000100019 \& \operatorname{lng}=e s}$

24. Espina MP. Desigualdad y política social en Cuba hoy. Conference "The Future of Social J ustice in Cuba". Panel "Social J ustice in Cuba: Assessment of Current Situation", May 27-29. Bellagio: Conference Center; 2008.

25. Espina MP. Desarrollo, desigualdad y políticas sociales. Acercamientos desde una perspectiva compleja. La Habana: Publicaciones Acuario, Centro Félix Varela; 2010.

http://scielo.sld.cu 
26. Bacallao J. Indicadores basados en la noción de entropía para la medición de las desigualdades sociales en salud. Rev Cubana Salud Pública [Internet]. 2007 Dic [citado 26 Feb 2011]; 33(4). Disponible en:

http://scielo.sld.cu/scielo.php?script=sci_arttext\&pid=S0864-

34662007000400007\&lng=es

27. Konings G, Harper S, Lynch J. Analysis of socioeconomic health inequalities using the concentration index. Int J Public Health. 2010;55:71-4.

28. Fraley C, Raftery AE. Model-based clustering, discriminant analysis and density estimation. JASA. 2002; 97:611-2.

29. Scanlan JP. Can we actually measure health disparities? Chance. 2006; 29:47-51.

30. Bacallao J. Dos temas polémicos en el contexto de la medición de las

desigualdades sociales en salud. Rev Cubana Salud pública [Internet]. 2007 [citado 18 Feb 2011]; 33(3). Disponible en:

http://scielo.sld.cu/scielo.php?script=sci_issuetoc\&pid $=0864-$

$\underline{346620070003 \& \operatorname{lng}=e s \& n r m=i s o}$

31. Halfon N, Larson K, Russ S. Why social determinants? Healthcare Quarterly. 2010; 14: 9-20.

32. Davidson KW, Pickering TG. Toward a causal model of cardiovascular responses to stress and the development of cardiovascular disease. Psychosomatic Med.

2003; 65: 9-21.

33. Programa de las Naciones Unidad para el Desarrollo (PNUD). Informe Regional sobre Desarrollo Humano para América Latina y el Caribe 2010. Actuar sobre el futuro: romper la transmisión intergeneracional de la desigualdad. Costa Rica: Ed. San José, PNUD; 2010.

* En 46 familias del total de 840 estudiadas en 12 consultorios de 6 áreas de salud, algunas de las variables estaban ausente, por lo que el análisis de conglomerados basado en modelos se concentró en las 794 familias con datos completos.

Recibido: 11 de mayo de 2012.

Aprobado: 29 de junio de 2012.

J orge Bacallao Gallestey. Centro de Investigaciones y Referencia de Aterosclerosis de La Habana (CIRAH). Policlínico "19 de Abril". Calle Tulipán y Panorama. Nuevo Vedado, Plaza. La Habana, Cuba.

Correo electrónico: bacallao@infomed.sld.cu

http://scielo.sld.cu 